\title{
Users, Economics, Technology: Unavoidable Interdynamics
}

\author{
Rui L. Aguiar · Ertan Onur • Knud Erik Skouby • \\ Mari Carmen Aguayo-Torres • \\ Thomas Skjødeberg Toftegaard
}

Published online: 16 March 2010

(c) The Author(s) 2010. This article is published with open access at Springerlink.com

\begin{abstract}
This paper briefly presents some conclusions of a brainstorming session on the way technology is evolving in ICT. Technology advances have overcome society ability to answer, both in economic and in human aspects. The current design paradigms, of agnostic technology development, need to be reconsidered, and the user needs to be repositioned at the center of future developments.
\end{abstract}

Keywords Technology evolution $\cdot$ Society and multidisciplinary approach

\section{Introduction}

It is nowadays an increasingly recognized reality that technology excellence per itself does not necessarily mandate technology evolution. Users and the overall society (including economics) play an increasing role in the success of specific technologies. In this dynamic

R. L. Aguiar $(\varangle)$

I.T., Universidade de Aveiro, Aveiro, Portugal

e-mail: ruilaa@ua.pt

E. Onur

Delft University of Technology, Delft, The Netherlands

e-mail: e.onur@tudelft.nl

K. E. Skouby

CTIF, Copenhagen, Denmark

e-mail: skouby@cmi.aau.dk

M. C. Aguayo-Torres

University of Malaga, Malaga, Spain

e-mail: aguayo@ic.uma.es

T. S. Toftegaard

A.S.E., Århus University, Århus, Denmark

e-mail: tst@cs.au.dk 
environment we are now living, users, technology and economics interplay with increasingly complex relations.

Society is changing fast, but the pace of technology is much faster than the change society is able to accept. Furthermore, society changes often in hard to predict ways, reacting to novel technologies in unexpected ways. User concerns are not the same today than we could see a couple of years ago-e.g., concerns with privacy, and privacy-aware technologies, are a recent trend. The relevance society pays to different concerns changes with time.

Recently, the user has taken an increasing important role in the ICT world. From a passive user, consuming services provided by operators, we now witness a user establishing its own interest communities, and providing its own added value to the communication process. The user becomes an actor in this economic system. Furthermore, with increasing competition, the user becomes a more valued asset, able to move between a wider choice of services.

The overall interplay between users, technology and economics is thus an issue that we must start to consider in our visions of technology evolution.

\section{User-Inspired R\&D}

Until now research has been focused on optimizing the communication technology from the point of view of the big corporation. However, innovation in large companies does not proceed now at the pace that was usual several years ago. Economic pressures and the increasingly multidisciplinary requirements in innovation, have mostly stopped disruptive innovation inside corporations. Innovation is coming from smaller companies, often recent start-ups, which are able to present novel ideas and added value - and then acquired by larger communication companies.

This change in development paradigm affected the interdynamics between economics and technology. It is no longer the big economic interests that drive technology development, but small companies, with radical new ideas. The success of Google and Facebook, to name just a few, are good examples of small companies that change the way technology is used.

These small companies are much more near the end-user. The trend is now on userinspired research, in the context of different application domains: iHealth, iEnergy, iHouse, etc... Research became interdisciplinary, with small teams bringing different competences to define new technologies and products: sensors, wireless infrastructure, signal and image processing, photonics, protocols, software-all these areas are brought together for different applications (See Fig. 1).

The interdynamics between users, technology and economics also have a different vector: how to move this user-inspired research into mainstream technology. There are clear differences between technology development and technology transfer. There are political pressures for technology transfer, until now biased towards large companies. Small companies are not able to influence political decisions, which impair as well the coming of disruptive ideas into the market. This is particularly crucial as innovation now happens along the lines of convergence, bridging together different communication worlds (e.g., mobile, multimedia, voice) - currently under the overview of different regulatory entities. Technology evolution, even if increasingly centred on the user view, cannot succeed without the best compromise between these interdynamics with economics and political forces. Unfortunately, these interdynamics are not linear, and often lead to unpredictable results, defining the fate of technical innovation.

A recent trend, with still unclear economic impacts, is the movement into Open Innovation [1]. Some companies are opening their patent pool for further developments, in specific 


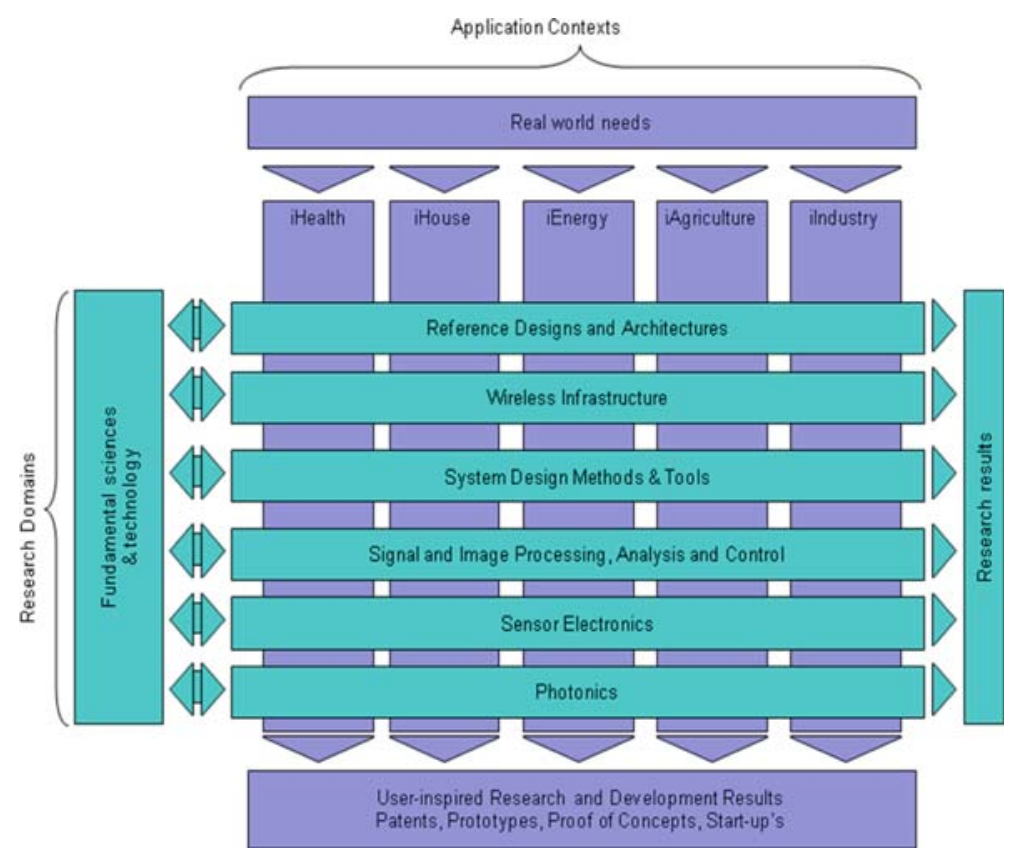

Fig. 1 A new research paradigm

application domains (even in areas such as rare diseases for the third world). The Internet development model follows this trend, which is a clear departure from traditional telecommunication development models. Nevertheless, the fast innovation pace possible with current technologies seems to be more appropriate to these open models than to the older controlled evolution models.

\section{Can Technology Support These Interdynamics?}

The existence of these interdynamics makes successful technology development unpredictable. The question arises, if we can use technology itself to develop solutions able to withstand whatever outcomes from this interplay.

Some trends on the evolution of complexity in technology are already apparent. Kurzweil [2] has already predicted that technology will overtake the human brain. By 2050 we should be able to have the equivalent intelligence of the human race in silicon. Is it possible for us to design technology that may be resilient to the interdynamics between users, economics and innovation. The ideal would be to develop a set of technologies that observe the environment (in all dimensions), develop its own strategies, and decides on its parameters-a continuous adaptation to society needs. This trend can now be already observed in technologies such as Software Defined Radio, or Cognitive Networks. The basic technology can evolve and adapt to regulation and market needs.

The dynamic scenarios now expected in wireless communications require this sort of adaptability. With ubiquitous environments around the corner, devices are expected to become multi-interface, multi-technology. Intelligent Spectrum Management, virtualised cooperative and distributed, associated with cognitive radio and cognitive networks concepts are 
becoming a common vision for the future. Virtualization abstracts the technological implementation details and complexity of the underlying devices and networks from the user. It helps all constituents emerge as a single virtual device providing seamless services to the user and enables users to offer and provide services. In this approach, energy-efficiency, resource utilization, and dependability requirements can be addressed in a modular way and backward-compatibility (in the sense of supporting existing technologies) can be provided. In this light, virtualization enables service provisioning by the end-users, supporting application/service portability and compatibility. When the users are enabled to provide services, they become prosumers-producers while consuming as well. In the end, we will expect these concepts to adapt to the society itself, to the user social structure, his communities.

It is now possible to envisage such a dynamic environment, with communities that integrate user needs into technology solutions [3]. A reasoning layer is developed that supports the interplay between user needs and technology parameterization-resorting to doctrines, and defining rules and attributes for managing technology. Such an approach can provide a rich and versatile set of solutions, able to adapt and respond to changes in the economic, regulatory and social environments.

Several questions exist, though. If such a technology set of solutions is pursued, then clear regulations on its usage have to be set, in such a way that stable, reliable environments are deployed. Also the overall economic motivation for such technologies must be clear, since its own flexibility leads to easier customer movement.

\section{Conclusions}

In this paper we opened the discussion on the issues associated with technology development. In particular, we discussed the increased interdynamics between users, technology and society. It is now apparent that is required more than technology excellence for the uptake of novel technologies. There are many issues surrounding the technical workspace that may impact the success of a given technology, and R\&D should be aware of this. Market reaction, regulatory frameworks, political support are all aspects that R\&D engineers should be aware.

It is increasingly worth to focus on user-centric research, pursuing multidisciplinary solutions that provide added value to specific application areas. It is also clear that technologies for dynamic environments are a trend for the future. The technology will be able to parameterize itself also in function of non-technical parameters. These trends provide some suggestions on how R\&D can proceed with enough flexibility to adapt itself to the variable outcomes of the society response to technology advances.

Open Access This article is distributed under the terms of the Creative Commons Attribution Noncommercial License which permits any noncommercial use, distribution, and reproduction in any medium, provided the original author(s) and source are credited.

\section{References}

1. Chesbrough, H. W. (2003). Open innovation: The new imperative for creating and profiting from technology. Boston: Harvard Business School Press.

2. Kurzweil, R. (2005). The singularity is near: When humans transcend biology. New York: Penguin.

3. Antoniadis, P., Le Grand, B., Satsiou, A., Tassiulas, L., Aguiar, R., \& Paulo Barraca, J., et al. (2008). Community building over neighbourhood wireless mesh networks. IEEE Technology and Society Magazine, 27(1), 48-56. 


\section{Author Biographies}

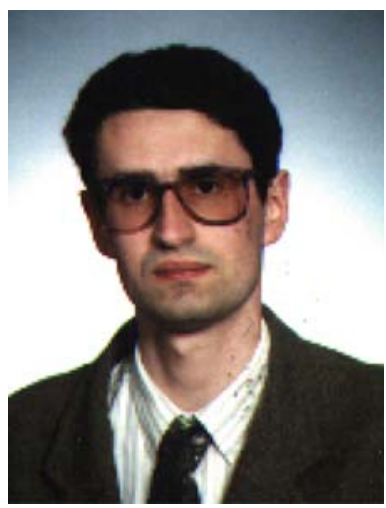

Rui L. Aguiar concluded his Ph.D. at the University of Aveiro, Portugal, in 2001. He is currently a associate professor at the Universidade de Aveiro and a researcher at Instituto de Telecomunicações, where he is leading a group on heterogeneous communications. Prof. Aguiar is an adjunct professor at INI, Carnegie Mellon. He is further coordinating the area of Networking and Multimedia. His current main interests lie in communication systems, focusing especially in wireless network evolutions. He has published more than 250 papers in national and international Journals and conferences in telecommunications systems and networks. Prof. Aguiar has acted as an expert evaluator in several countries. He has also taken key technical coordination responsibilities in several national and European projects and has been active in the technical committee of many conferences. He has been TPC Co-Chair of ICNS'05 and ISCC'07, and General Chair of ICT2006. He is a chartered Engineer, a Senior Member of IEEE and a member of ACM.

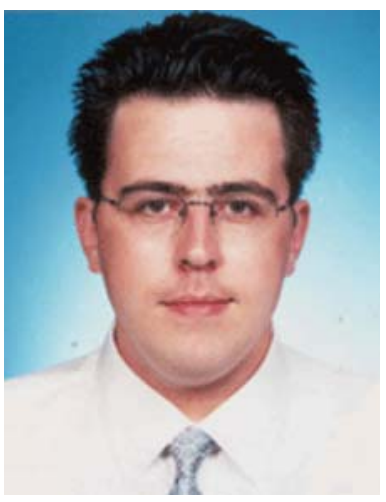

Ertan Onur received the B.S. degree in computer engineering from Ege University, Izmir, Turkey in 1997, and the M.S. and Ph.D. degrees in computer engineering from Bogazici University, Istanbul, Turkey in 2001 and 2007, respectively. Dr. Onur is a BAL'93 graduate. After the B.S. degree, he worked for LMS Durability Technologies GmbH, Kaiserslautern, Germany. During the M.Sc. and Ph.D. degree, he worked as a project leader at Global Bilgi, Istanbul and as an R\&D project manager at Argela Technologies, Istanbul. Presently, he is an assistant professor at EEMCS, WMC, Delft University of Technology, Netherlands. Dr. Onur's research interests are in the area of computer networks, personal networks, and wireless and sensor networks. He is a member of IEEE.

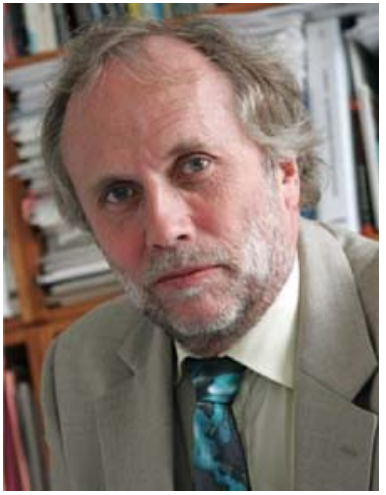

Knud Erik Skouby is professor and founding director of center for Communication, Media and Information technologies (CMI), Aalborg University/Copenhagen - a center providing a focal point for multidisciplinary research and training in applications of CMI-as part of the international research network CTIF. Has a career within consultancy and as a university teacher since 1972 . In the last 20 years he has worked in telecoms, focusing on mobile/wireless: Techno-economic Analyses; Development of mobile/wireless applications and services: Regulation of telecommunications Chair of WG1 in WWRF-Wireless World Research Forum-a forum for pre-standardization activities. He has participated as a project manager and partner in a number of international (incl. a long term activity in Africa), European and Danish research projects. He has served on a number of public committees within the areas of telecom, IT and broadcasting; as a member of boards of professional societies; as a member of organizing boards, evaluation committees and as invited speaker on international conferences; published a number of Danish and international articles, books and conference proceedings in the areas of convergence, mobile/wireless development, telecommunications regulation, technology assessment (information technology and telecommunications), demand forecasting and political economy. 

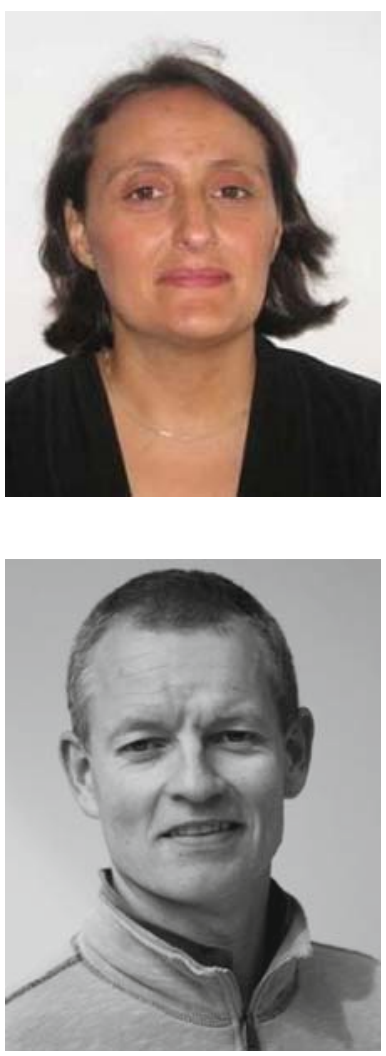

Mari Carmen Aguayo-Torres received the M.S. and Ph.D. degrees in Telecommunication Engineering from the University of Málaga, Spain, in 1994 and 2001, respectively. Currently, she is working at the Department of Communications Engineering, at the same university. Her main research interests include probabilistic QoS guarantees for wireless communications, end-to-end QoS over heterogeneous networks, crosslayer design, multiuser OFDM, and adaptive modulation and coding for fading channels.
Thomas Skjødeberg Toftegaard (former Thomas Toftegaard Nielsen) holds a M.Sc.E.E. (1995) and a Ph.D. (1999) in wireless communications from Aalborg University, Denmark. April 1, 2009 he was appointed Professor in Communication Technology at Aarhus School of Engineering, Aarhus University, Denmark. Additionally he serves as Director of the Electrical Engineering and Information \& Communication Technology at Engineering College of Aarhus. He is chairman of the study committee of the M.Sc.E.E. and ICT at Aarhus School of Engineering. As Professor he is leading a R\&D group on Next Generation Wireless Infrastructure focusing on seamless connectivity. His main research interests is on future intelligent wireless connectivity, working with mobile communications, sensor networks, massive dense network architectures, network complexity, wireless IP, software defined radio, cognitive radio and ubiquitous wireless networks. In addition to academic work, his professional carrier includes a $14+$ year tenure in the high-tech industry in the Scandinavian region. With responsibilities exclusively in the $\mathrm{R} \& \mathrm{D}$, project management and product development domains, he has developed expertise in the design and development of mobile communication systems, mobile phones and product components. He has more than 10 years of experience in leading a R\&D unit in a global organization build while working for the mobile operator Sonofon (now Telenor), Nokia Telecommunications, L. M. Ericsson and Tieto. Professor Toftegaard has published one book on wireless communications, as well as a number of journal and conference papers. 